\title{
SYNCHROTRON RADIATION FROM ENERGETIC ELECTRONS EMITTED BY AGN: A PROBE FOR MAGNETIC FIELDS IN EXTERNAL GALAXIES
}

\author{
MATTHEW G. BARING \\ Max Planck Institut für Astrophysik \\ Karl Schwarzschild Strasse 1 \\ D-8046 Garching bei München, West Germany
}

ABSTRACT: Shock acceleration of protons in the central region of active galaxies can energize them to Lorentz factors as high as $10^{8}$ (Sikora et al., 1987), and these can subsequently produce a host of other relativistic particles including pions, $e^{+} e^{-}$pairs and neutrons. The luminosities of each species are expected to be of the same order of magnitude. Rapid decay of the pions leads to the secondary production of photons and pairs with energies of around $10^{9}-10^{11} \mathrm{MeV}$. The electrons and positrons can escape the compact central region and interact with the microwave background forming a pair cascade, and can also emit synchrotron radiation in the magnetic field. The neutrons do not interact with the field, and a significant fraction of them can escape the central region of a galaxy (Kirk and Mastichiadis, 1989). They can travel until they decay, producing protons and electrons in outer regions of the galaxy. Their decay time of $\gamma_{n} \tau_{n}$ gives a typical length for decay of about $1 \mathrm{kpc}$ for the most energetic neutrons. The synchrotron radiation of these decay product electrons is examined in Baring (1989, in preparation), and it produces definite signatures of galactic magnetic fields. Magnetic fields of $1 \mu G$ imply synchrotron emission in the X-ray and soft gamma-ray range for maximum Lorentz factors of $\gamma_{e}=10^{10}$, with a continuum extending down to much lower energies. It is observed that cooler neutrons deposit electrons at smaller radii, and these electrons are cooler (in a decay $\gamma_{e} \sim \gamma_{n}$ ). Hence the radiation would be cooler at smaller radii. This provides a diagnostic for the magnetic field: estimates of the field strength are possible from cut-offs that are expected in spectra from galactic halos. The injection of energetic electrons via neutron decay is found to yield a sharp cut-off in the injection distribution at $\gamma_{m}=\gamma_{e} \sim r / \tau_{n} c$ at radius $r$. Below this, no electrons are injected since they are produced in decays at smaller radii. This implies a low energy cutoff of $\omega_{m}=\gamma_{m}^{2} B m_{e} c^{2}$ in the spectrum at given radius. Typically for $r=10 \mathrm{pc}$ and a field of $1 \mu G$, the cutoff is at $10^{-8} m_{e} c^{2}$ in the far infra-red. At larger radii, this low energy cutoff rapidly increases to X-ray energies. This cut-off provides a good way to measure the magnetic field strength and obtain its spatial dependence. In practice the situation is complicated by the superposition of different regions within the galaxy along the line of sight. Ways in which the observations could be deconvolved are discussed in Baring (1989, in preparation). A real possibility exists for detecting these signatures of energetic neutron emission from central regions of nearby galaxies and using the electron synchrotron spectra to spatially map galactic fields.

\section{REFERENCES:}

Kirk, J. G., and Mastichiadis, A., 1989 Astron. Astrophys. 213 , 75

Sikora, M., et al., 1987 Astrophys. J. (Letters) 320 , L81 